\title{
Modeling the ocean effect of geomagnetic storms
}

\author{
N. Olsen and A. Kuvshinov \\ Danish Center of Planetary Science/Danish Space Research Institute, Juliane Maries Vej 30, DK-2100 Copenhagen, Denmark
}

(Received December 8, 2003; Revised March 25, 2004; Accepted March 31, 2004)

\begin{abstract}
At coastal sites, geomagnetic variations for periods shorter than a few days are strongly distorted by the conductivity of the nearby sea-water. This phenomena, known as the ocean (or coast) effect, is strongest in the magnetic vertical component. We demonstrate the ability to predict the ocean effect of geomagnetic storms at geomagnetic observatories. The space-time structure of the storm is derived from the horizontal components at worldwide distributed observatories from which we predict the vertical component using a model of the Earth's conductivity that a) only depends on depth, and b) includes the conductivity of the sea-water. The results for several strong geomagnetic storms (including the "Bastille Day" event of July 14-15, 2000) show much better agreement (improvement by up to a factor of 2.5) between the observed and the modeled magnetic vertical component at coastal sites if the oceans are considered. Our analysis also indicates a significant local time asymmetry (i.e., contributions from spherical harmonics other than $P_{1}^{0}$ ), especially during the main phase of the storm.
\end{abstract}

Key words: Electromagnetic induction, ocean effect, geomagnetic storms.

\section{Introduction}

A significant portion of magnetic variations during geomagnetic storms is due to induced currents in the Earth's interior. External and internal parts impact on the horizontal $(X, Y)$ and vertical $(Z)$ components differently, and the analysis of both allows for a separation of external and internal parts according to C. F. Gauss. Alternatively, the separation can be done using only the horizontal components and a model of the Earth's electrical conductivity. This allows for a prediction of $Z$ from $(X, Y)$, and the comparison of simulated and observed $Z$ can be used to test the model.

It is well known that geomagnetic variations at coastal sites are strongly distorted by the conductivity of the nearby sea-water for periods up to a few days, a phenomena called ocean or coast effect (Parkinson and Jones, 1979; Fainberg, 1980; Parkinson, 1983; Rikitake and Honkura, 1985; Parkinson, 1987). In a previous paper (Kuvshinov et al., 2002b) we demonstrated the capability to correct electromagnetic response functions at coastal sites for induction in the oceans. The present paper concerns the distorting effect of the oceans on the time series of the magnetic field (rather than response functions) and investigates the possibility of modeling the ocean effect of magnetic storms.

To first order, geomagnetic storms can be described by an intensification of the (westward directed) magnetospheric ring-current (e.g., Rostoker et al., 1997). The time change of the external current system sets up a corresponding internal current system of reversed sign, and hence the major part of induced currents during storms are eastward directed. While a significant part of the induced currents flow in the open oceans (and in the underlying mantle), coastlines will

Copy right (c) The Society of Geomagnetism and Earth, Planetary and Space Sciences (SGEPSS); The Seismological Society of Japan; The Volcanological Society of Japan; The Geodetic Society of Japan; The Japanese Society for Planetary Sciences; TERRAPUB. force them to deviate from the West-East geometry dictated by the external (inducing) currents. This leads to current channeling, which is especially pronounced at the edges of continental barriers like Southern Africa. We investigate this by predicting the storm-time variation in $Z$ from that in $X$ and $Y$ using a) a one-dimensional (1-D) model of the Earth's conductivity, and b) a three-dimensional (3-D) conductivity model that includes the high conductivity of the sea-water.

\section{Global Description of Storm-Time Variations}

At the Earth's surface, the magnetic field of geomagnetic storms can be derived from a scalar magnetic potential $V$. The space-time structure of the potential describing the external, inducing, current contributions is expanded according to

$$
V^{(e)}(t, r, \theta, \phi)=a \operatorname{Re}\left\{\sum_{k=-\infty}^{\infty} \sum_{n=1}^{\infty} \sum_{m=0}^{n} \epsilon_{n}^{m}\left(\frac{r}{a}\right)^{n} Y_{n}^{m} e^{i \omega_{k} t}\right\}
$$

where $\epsilon_{n}^{m}\left(\omega_{k}\right)$ are the (complex) expansion coefficients of the external part of the potential at frequency $\omega_{k},(r, \theta, \phi)$ are spherical coordinates with $r=a=6371.2 \mathrm{~km}$ denoting the mean Earth's radius, $\theta$ and $\phi$ geomagnetic colatitude and longitude, and $Y_{n}^{m}(\theta, \phi)=P_{n}^{m}(\cos \theta) e^{i m \phi}$ where $P_{n}^{m}$ are the associated Legendre functions. $\operatorname{Re}\{\cdot\}$ denotes the real part. In practice the summation of Eq. (1) is finite: the number of frequencies is determined by the length of the times series (here 240 hourly mean values are used, resulting in 120 frequencies), and the spherical harmonic expansion is performed up to $n=3$.

A similar expansion is possible for the internal, induced, part $V^{(i)}$ of the potential with $\iota_{n}^{m}(\omega)$ being the corresponding expansion coefficients. The magnetic variation follows as $\delta \mathbf{B}=-\operatorname{Re}\{\operatorname{grad} V\}$ where $V=V^{(e)}+V^{(i)}$. 
If the electrical conductivity $\sigma$ of the Earth's interior is 1D (i.e. spherically symmetric, $\sigma(r)$ depends only on depth), each external coefficient $\epsilon_{n}^{m}$ induces only one internal coefficient $\iota_{n}^{m}$ (of the same degree $n$ and order $m$ ), and their ratio $Q$ is independent of $m$ (Schmucker, 1985a)

$$
\iota_{n}^{m}(\omega)=Q_{n}(\omega) \epsilon_{n}^{m}(\omega) .
$$

In this case, the combined potential $V=V^{(e)}+V^{(i)}$ (for a fixed frequency $\omega_{k}$ ) can be written as

$$
V_{1 \mathrm{D}}=a \sum_{n=1}^{3} \sum_{m=0}^{n} \epsilon_{n}^{m}\left[\left(\frac{r}{a}\right)^{n}+Q_{n}\left(\frac{a}{r}\right)^{n+1}\right] Y_{n}^{m} .
$$

However, in the general case of a 3-D conductivity ( $\sigma$ depends on vertical and horizontal coordinates), each external coefficient $\epsilon_{n}^{m}$ induces a whole spectrum of internal coefficients $\iota_{n}^{m}$, and Eq. (2) has to be replaced with the more general relationship (Olsen, 1999)

$$
\iota_{n}^{m}(\omega)=\sum_{l, k} q_{l n}^{k m}(\omega) \epsilon_{l}^{k}(\omega) .
$$

The main sources of geomagnetic storm variations are large-scale (at least at non-polar latitudes), and therefore external coefficients $\epsilon_{n}^{m}$ of relatively low $n$ and $m(\leq 3)$ are sufficient to describe their spatial behavior. The induced field, however, is strongly influenced by local conductivity anomalies, for instance near coastlines, and therefore a large number of internal coefficients $\iota_{n}^{m}$ are required for modeling the induced field. Instead of performing an explicit expansion of the internal field, we determined the response $R_{n}^{m}(\omega, r, \theta, \phi)$ of an inducing field given by the spherical harmonic $Y_{n}^{m}$ at frequency $\omega$ using the approach described in the next section (for a 1-D conductivity $R_{n}^{m}(\omega, r, \theta, \phi)=$ $\left.Q_{n}(\omega)(a / r)^{n+1} Y_{n}^{m}(\theta, \phi)\right)$. This allows us to write the combined potential of the $3-\mathrm{D}$ case as

$$
V_{3 \mathrm{D}}=a \sum_{n=1}^{3} \sum_{m=0}^{n} \epsilon_{n}^{m}\left[\left(\frac{r}{a}\right)^{n} Y_{n}^{m}+R_{n}^{m}\right] .
$$

The magnetic field variations are derived from the potential $V$ of Eq. (3) or (5) according to $\delta X=-\delta B_{\theta}=$ $\operatorname{Re}\{\partial V / \partial \theta\} / r, \delta Y=+\delta B_{\phi}=-\operatorname{Re}\{\partial V / \partial \phi\} /(r \sin \theta)$, $\delta Z=-\delta B_{r}=\operatorname{Re}\{\partial V / \partial r\}$.

\section{Simulation of Induction in the Oceans}

Electromagnetic induction simulations require a model of the electrical conductivity of the Earth's interior. Our 3D model consists of a thin spherical shell of conductance $S(\theta, \varphi)$ at the Earth's surface with a radially symmetric spherical conductivity $\sigma(r)$ underneath. A realistic model of the shell conductance $S(\theta, \varphi)$ is obtained by considering contributions both from sea-water and from sediments.

The conductance of the sea water has been derived from the global $5^{\prime} \times 5^{\prime}$ NOAA ETOPO map of bathymetry/topography, multiplying the water depth by a mean seawater conductivity. Note that the seawater conductivity varies between 3 and $4 \mathrm{~S} / \mathrm{m}$, depending for example on salinity and temperature. We used a mean value of 3.2 $\mathrm{S} / \mathrm{m}$; the errors introduced by deviations from that value are believed to be smaller than those due to insufficient knowledge of mantle conductivity. The conductance of the sediments (for oceanic and continental regions) has been derived from the global sediment thicknesses given by the $1^{\circ} \times 1^{\circ}$ map of Laske and Masters (1997) by using a heuristic procedure similar to that of Everett et al. (2003). In general the sediments contribute with up to $10 \%$ to the total surface conductance. However, in areas such as the Gulf of Mexico, Arctic Ocean, Black and Caspian Seas, the conductance of the accumulated sediments is comparable to that of the sea water. Figure 1 shows the conductance of this surface shell. The underlying conductivity $\sigma(r)$ is compiled from the four-layer model of Schmucker (1985b) for depths greater than $100 \mathrm{~km}\left(0.014 \mathrm{Sm}^{-1}\right.$ between 100 and $500 \mathrm{~km}, 0.062 \mathrm{Sm}^{-1}$ between 500 and $750 \mathrm{~km}$, and $2.4 \mathrm{Sm}^{-1}$ at depths greater than $750 \mathrm{~km}$ ) whereas for the upper $100 \mathrm{~km}$ we take $\sigma=3 \cdot 10^{-4} \mathrm{Sm}^{-1}$. For our 1-D model results we replaced the surface shell $S(\theta, \phi)$ by a shell of constant mean continental conductance $\bar{S}=400 \mathrm{~S}$. Maxwell's equations are solved numerically according to the scheme presented in Kuvshinov et al. (2002a) on a mesh with spatial resolution of $1^{\circ} \times 1^{\circ}$ in the frequency domain (for 20 logarithmically spaced frequencies between $1 / 10$ days $^{-1}$ and $1 / 2 \mathrm{hrs}^{-1}$ ). Finally, we compute $R_{n}^{m}\left(\omega, \theta_{j}, \phi_{j}\right)$ (and its derivatives wrt. $\theta$ and $\phi$ needed for computing $\delta X$ and $\delta Y$ ) for $n, m \leq 3$ and for the locations $\left(\theta_{j}, \phi_{j}\right)$ of the observatories used in this study.

\section{Data Analysis}

We have applied our method to several major geomagnetic storms (Dst index exceeds $-200 \mathrm{nT}$ ) of the last years. First, we have investigated the geomagnetic storm on July 14-15, 2000 ("Bastille Day") which was one of the largest in recent years; Dst dropped to -300 nT. We used hourly mean values of observatories equatorward of $\pm 60^{\circ}$ geomagnetic latitude, the distribution of which is shown in Fig. 1. A time segment of 10 days length (starting on July 13, 00:00 UT) for each component and each observatory is Fourier transformed after subtracting a baseline defined by the night-time level of nearby quiet days. The horizontal components were rotated from geographic to geomagnetic components. A robust spherical harmonic analysis of the horizontal components was performed for each frequency both for the 1-D case (Eq. (3)) and the 3-D case (Eq. (5)), and the expansion coefficients $\epsilon_{n}^{m}(\omega)$ for $n, m \leq 3$ were estimated for 120 frequencies between $1 / 240 \mathrm{hrs}^{-1}$ and $1 / 2 \mathrm{hrs}^{-1}$ (the interpolation of the responses $R_{n}^{m}(\omega)$ from the 20 frequencies of the simulation to the actual 120 frequencies of the data analysis was done with splines). Finally, time series of synthetic magnetic variations $\delta X, \delta Y$ and $\delta Z$ were computed for all observatories for the 1-D and 3-D case, respectively, and were compared with the observed time series. Other major recent storms were analyzed in a similar way.

\section{Results and Discussion}

Figure 2 shows the time series of real (blue) and imaginary (red) part of the external expansion coefficients $\epsilon_{n}^{m}(t)$ for the Bastille-Day event (July 14-15, 2000). The coefficients are for the 3-D case and are obtained by Fourier transforming $\epsilon_{n}^{m}(\omega)$.

Since the external expansion coefficients obtained using 


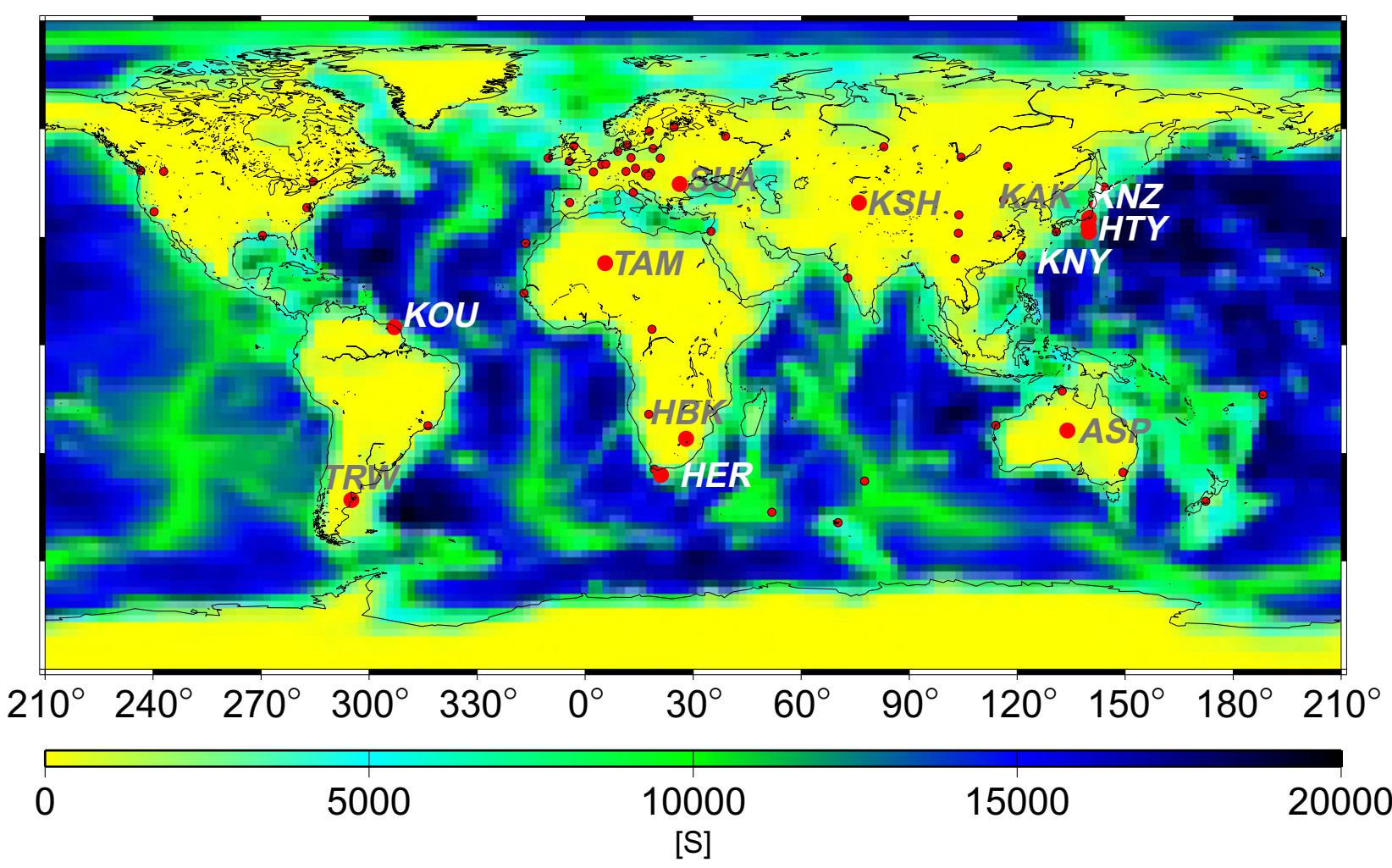

Fig. 1. Conductance of the surface shell describing oceans and sediments. Also shown are the locations of the observatories used in this study (small red dots) and of those used in Fig. 3 (large red dots).
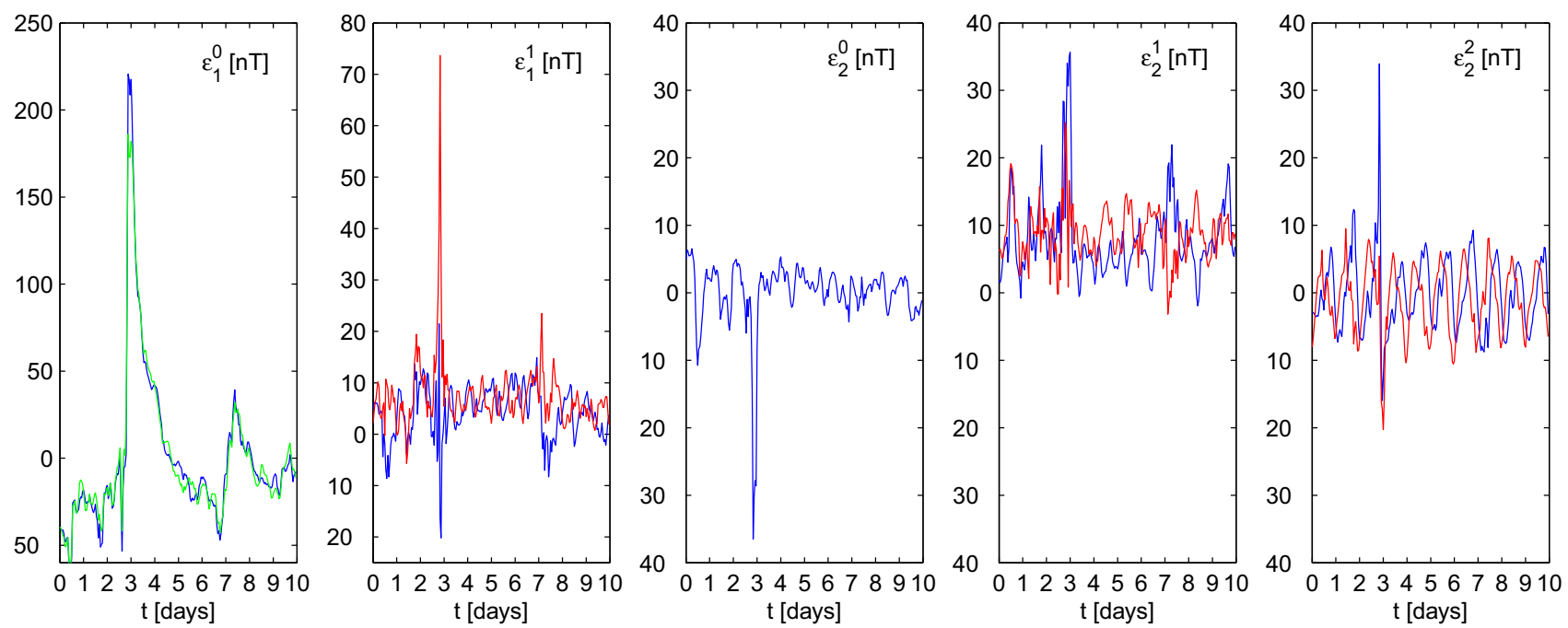

Fig. 2. Time series of real (blue) and imaginary (red) parts of the external spherical harmonic expansion coefficients $\epsilon_{n}^{m}$, in nT. The green line presents an estimate of $\epsilon_{1}^{0}$ based on the Dst-index (Eq. (6)). $t=0$ corresponds to July 13, 2000, 00:00 UT.

the 1-D and 3-D conductivity models are very similar, we only present the results of the 3-D model. As expected, the main contribution during the storm is described by the coefficient $\epsilon_{1}^{0}$. The green line shows another estimate of that coefficient based on the $D s t$-index,

$$
\epsilon_{1}^{0}=-D s t /\left(1+\widetilde{Q}_{1}\right)
$$

the factor $\left(1+\widetilde{Q}_{1}\right)$ with $\widetilde{Q}=0.27$ (found from satellite data (Langel and Estes, 1985) and corresponding to an infinite conductor at $1200 \mathrm{~km}$ depth, overlaid by an insulating mantle) is a first order correction of induction effects. $\epsilon_{1}^{0}$ describes the magnetic effect of a symmetric magnetospheric ring current in the geomagnetic equatorial plane. There is good agreement between the results of the two approaches to estimate $\epsilon_{1}^{0}$; differences are probably mostly due to the fact that we used data from up to 65 non-polar observatories, whereas Dst is estimated using only four observatories. However, Figure 2 shows that a significant contribution (up to $30 \%$ ) is due to other coefficients, for instance $\epsilon_{1}^{1}$, especially during the main phase of the storm. This is in agreement with previous investigations (Fujii and Schultz, 2002; 

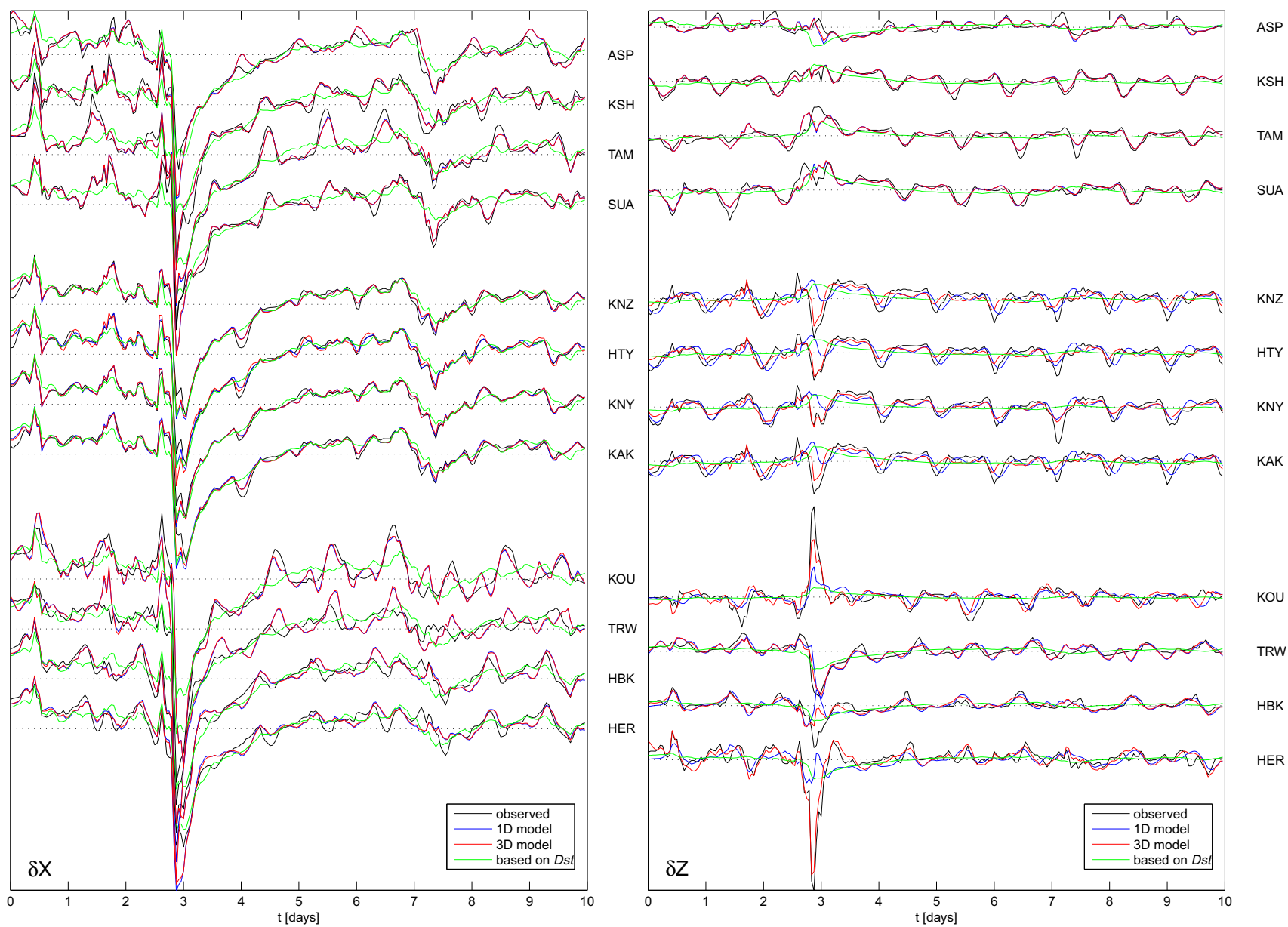

Fig. 3. Time series of observed and modeled $\delta X$ and $\delta Z$ (sum of external and induced contributions), respectively, at selected observatories. 3-D model results are shown in red, 1-D results in blue and observed fields in black. The green lines present values based on the $D s t$-index. $t=0$ corresponds to July 13, 2000, 00:00 UT. 100 nT offset between the zero-levels (dashed lines).

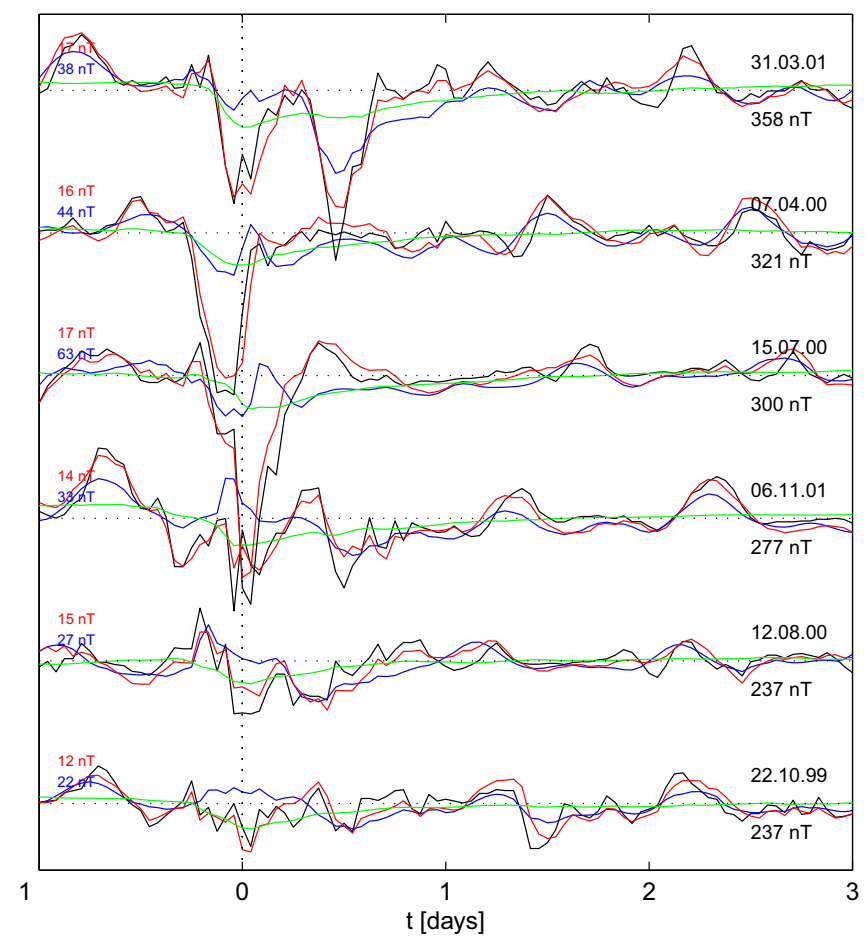

Fig. 4. Time series of observed (black) and modeled $\delta Z$, respectively, at Hermanus for selected geomagnetic storms. 3-D model results are shown in red, 1 -D results in blue, and values based on the $D s t$-index in green. The storms have been sorted according to the peak value of $D s t$ (which defines $t=0$, vertical dashed line), the value of which, together with the date, is shown on the right side of the figure. The numbers at the left present rms deviations (see text). $150 \mathrm{nT}$ offset between the zero-levels (horizontal dashed line) of each storm. 
Olsen, 1998) who pointed out that approximating the geomagnetic field variations by a $P_{1}^{0}$ source may lead to biased responses, and therefore the contribution of higher degree spherical harmonics can be important. The diurnal variation of the coefficient $\epsilon_{2}^{0}$ reflects the daily shift of the (symmetric) ring-current northward (or southward) of the equatorial plane, due to the precession of the geomagnetic axis around the axis of rotation. No attempt has been made to remove $S q$, the regular daily variation of ionospheric origin. Hence it shows up in the higher terms, especially in $\epsilon_{2}^{1}$ (which is the largest expansion coefficient of $S q$ ). To first order, $S q$ is a local time phenomena (rather than a UT phenomena, as geomagnetic storms), and therefore real and imaginary part of $\epsilon_{1}^{1}$ and $\epsilon_{2}^{1}$ are out of phase, as expected for a local time phenomena.

Figure 3 presents time series of observed and modeled $\delta X$ (left) and $\delta Z$ (right) components at selected observatories. The results of the 3-D model calculations are shown in red, those of the 1-D model in blue, and the black lines present the observed fields. The green lines show values based on Dst (Eq. (6)). While 3-D and 1-D results are very similar in the horizontal component and agree with the observed values rather well, there are considerable differences between the 1$\mathrm{D}$ and the 3-D results in the vertical component for most of the displayed observatories. $S q$ shows up in the observed as well as in the 1-D and 3-D results (especially in $\delta Z$ ), but not in the values based on $D s t$ (since $S q$ has been removed when determining $D s t$ ). The largest difference between 1-D and 3-D model results is found at the south-African observatory Hermanus (HER); the peak of $\delta Z$ during the maximum of the storm is $-220 \mathrm{nT}$ for the $3-\mathrm{D}$ case, which is much closer to the observed value $(-250 \mathrm{nT})$ than the $1-\mathrm{D}$ result $(-50 \mathrm{nT})$. Hartebeesthoek (HBK), located $1250 \mathrm{~km}$ to the North-East of Hermanus, shows a much weaker ocean effect in $\delta Z$. However, also here the 3-D result is closer to the observed values than the $1-D$ result. The Argentinean observatory Trelew (TRW), located at similar geomagnetic latitude as Hermanus, does not show this anomalous behavior of $\delta Z$, due to its larger distance from the deep sea. However, Kourou (KOU) in French Guiana shows again large differences between the 1-D and 3-D results, and a closer agreement between the 3-D results and observations.

Also the Japanese observatories (KAK, KNY, HTY, KNZ) show a clear ocean effect, due to their proximity to a deepsea trench. Much better agreement between the observations and model results is obtained when the conductivity of the sea-water is considered.

For comparison, time series of $\delta X$ and $\delta Z$ at the four inland observatories ASP (in Central Australia), TAM (North Africa), SUA (Europe) and KSH (Central Eurasia) are also shown in the figure. As expected for locations far away from the coast, there is hardly any difference between the 1-D and 3-D modeling results.

In addition to conductivity changes due to the presence of sea-water, there might be discontinuities within the igneous crust and mantle associated with the continent-ocean boundary. Strictly speaking anomalous induction near coastlines has at least two possible contributions: ocean effect and the conductivity discontinuities within the crust and mantle (say, subduction slabs) specifically associated with continent-ocean boundaries. However, simulations using conductivity models with and without laterally inhomogeneous lithosphere and upper mantle at the continent-ocean transition indicate that the ocean effect is dominating (e.g., Kuvshinov et al., 2004).

We have applied our method to other major storms of the last years. Figure 4 presents the results for Hermanus. Although these storms are fairly similar in terms of the temporal behavior of $D s t$, the magnetic effect at an individual observatory differs. In addition to induction effects, contributions from higher harmonics are important, which is evident when comparing the storms of July 15, 2000 and Nov. 6, 2001. The predictions based on Dst (green curves) are very similar for both storms (since $D s t$ is similar). The 1-D model results (blue curves) differ, probably because anomalous induction is not taken into account (although contributions from spherical harmonics other than $P_{1}^{0}$ are considered). However, only the 3-D results (red) reproduce the observations (black). The superiority of the 3-D results is also evident when comparing the root mean squares (rms) deviation between observed and predicted $\delta Z$, calculated for the period 12 hours before until 24 hours after the peak in Dst. For the July 15 storm, the differences between observations and predictions based on the 1-D and the 3-D model are $\mathrm{rms}_{1 \mathrm{D}}=63 \mathrm{nT}$ and $\mathrm{rms}_{3 \mathrm{D}}=17 \mathrm{nT}$, respectively (cf. the numbers on the left side of the figure). The corresponding values for the storm of Nov. 6, 2001, are $\mathrm{rms}_{1 \mathrm{D}}=33 \mathrm{nT}$ and $\mathrm{rms}_{3 \mathrm{D}}=15 \mathrm{nT}$. Analyzing all major storms for the four years 1998-2001, we find a mean reduction of the residuals of a factor $\overline{\mathrm{rms}_{1 \mathrm{D}}} / \overline{\mathrm{rms}_{3 \mathrm{D}}}=2.5$ for Hermanus and a similar reduction for the Japanese observatories. This clearly demonstrates the ability of the presented method to model the ocean effect of geomagnetic storms.

\section{Conclusions}

We have presented a method for modeling the ocean effect of geomagnetic storms by solving the induction equation given the conductivity distribution of Earth's interior (that includes the conductivity of the sea-water) and the timespace structure of the storm (derived from the horizontal components of worldwide distributed observatories). The results for several major geomagnetic storms show much better agreement between the observed and the simulated magnetic vertical component at coastal sites when the oceans are considered and contributions from spherical harmonic terms other than $P_{1}^{0}$ are included. The latter indicates a significant asymmetry of the storm, especially during the main phase.

The results reported here are based on observatory data only. Work is ongoing to include high-precision magnetic satellite data (Ørsted, CHAMP, ... ) to investigate anomalous induction effects at satellite altitude. The results of this effort will be the subject of a forthcoming publication.

Acknowledgments. We wish to thank Mike Purucker and an anonymous referee for valuable suggestions on how to improve the manuscript.

\section{References}

Everett, M., S. Constable, and C. Constable, Effects of near-surface conductance on global satellite induction responses, Geophys. J. Int., 153, 
277-286, 2003.

Fainberg, E. B., Electromagnetic induction in the world ocean, Geophys. Surveys, 4, 157-171, 1980 .

Fujii, I. and A. Schultz, The 3D electromagnetic response of the Earth to ring current and auroral oval excitation, Geophys. J. Int., 151, 689-709, 2002.

Kuvshinov, A. V., D. B. Avdeev, O. V. Pankratov, S. A. Golyshev, and N. Olsen, Modelling electromagnetic fields in 3D spherical Earth using fast integral equation approach, in 3 D Electromagnetics, edited by M. S. Zhdanov and P. E. Wannamaker, chap. 3, pp. 43-54, Elsevier, Holland, 2002a.

Kuvshinov, A. V., N. Olsen, D. B. Avdeev, and O. V. Pankratov, Electromagnetic induction in the oceans and the anomalous behaviour of coastal $C$-responses for periods up to 20 days, Geophys. Res. Lett., 29, doi:10.1029/2001GL014,409, 2002b.

Kuvshinov, A. V., H. Utada, D. Avdeev, and T. Koyama, 3-D modelling and analysis of the Dst C-responses in the North Pacific ocean region, revisited, Geophys. J. Int., 2004 (in review).

Langel, R. A. and R. H. Estes, Large-scale, near-Earth magnetic fields from external sources and the corresponding induced internal field, J. Geophys. Res., 90, 2487-2494, 1985.

Laske, G. and G. Masters, A global digital map of sediment thickness, EOS Trans. AGU, 78, F483, 1997.

Olsen, N., Estimation of $C$-responses $(3 \mathrm{~h}$ to $720 \mathrm{~h}$ ) and the electrical conductivity of the mantle beneath Europe, Geophys. J. Int., 133, 298 308, 1998.
Olsen, N., Induction studies with satellite data, Surveys in Geophysics, 20, 309-340, 1999.

Parkinson, W. D., Introduction to Geomagnetism, Scottish Academic Press, Edinburgh, 1983.

Parkinson, W. D., The global conductivity distribution, Surveys in Geophysics, 9, 235-244, 1987.

Parkinson, W. D. and F. W. Jones, The geomagnetic coast effect, Rev. Geophys., 17, 1999-2015, 1979.

Rikitake, T. and Y. Honkura, Developments in Earth and planetary sciences, in Solid Earth Geomagnetism, edited by T. Rikitake, vol. 5, Terra Scientific Publishing Company, 1985.

Rostoker, G., E. Friedrich, and M. Dobbs, Physics of magnetic storms, in Magnetic Storms, edited by B. T. Tsurutani, W. D. Gonzales, Y. Kamide, and J. K. Arballo, vol. 98 of Geophysical Monograph Series, pp. 149160, American Geophysical Union, Washington DC, 1997.

Schmucker, U., Magnetic and electric fields due to electromagnetic induction by external sources, in Landolt-Börnstein, New-Series, 5/2b, pp. 100-125, Springer-Verlag, Berlin-Heidelberg, 1985a.

Schmucker, U., Electrical properties of the Earth's interior, in LandoltBörnstein, New-Series, 5/2b, pp. 370-397, Springer-Verlag, BerlinHeidelberg, 1985b.

N. Olsen (e-mail: nio@dsri.dk) and A. Kuvshinov 